\title{
Protée
}

\section{Zoom sur zone zoologique}

\section{Orna Ben-Meir}

Volume 27, numéro 1, 1999

\section{La Mort de Molière et des autres}

URI : https://id.erudit.org/iderudit/030544ar

DOI : https://doi.org/10.7202/030544ar

Aller au sommaire du numéro

Éditeur(s)

Département des arts et lettres - Université du Québec à Chicoutimi

ISSN

0300-3523 (imprimé)

1708-2307 (numérique)

Découvrir la revue

\section{Citer cet article}

Ben-Meir, O. (1999). Zoom sur zone zoologique. Protée, 27(1), 69-74. https://doi.org/10.7202/030544ar

\section{Résumé de l'article}

Cet article s'intéresse à deux personnages intrigants qui apparaissent dans la vidéo minimaliste de Robert Wilson, La Mort de Molière. Bien que le renard, joué par un acteur, et le scarabée, qui est un simple accessoire de théâtre, soient les seuls animaux présents comme personnages dans cette oeuvre, d'autres représentations animales peuvent être retrouvées : des « animaux acoustiques " sont animés par des effets sonores, des «animaux verbaux» sont mentionnés dans le texte de Müller. À la lumière de cet usage régulier d'un bestiaire animal dans l'oeuvre de Wilson, l'article explore les contextes visuels et verbaux de la symbolique animale présente dans La Mort de Molière, de façon entre autres à suggérer des pistes d'interprétation.
Ce document est protégé par la loi sur le droit d'auteur. L'utilisation des services d'Érudit (y compris la reproduction) est assujettie à sa politique d'utilisation que vous pouvez consulter en ligne.

https://apropos.erudit.org/fr/usagers/politique-dutilisation/ 


\section{ZOOM SUR ZONE ZOOLOGIQUE}

Traduit de I'anglais par Jean-Pierre Vidal

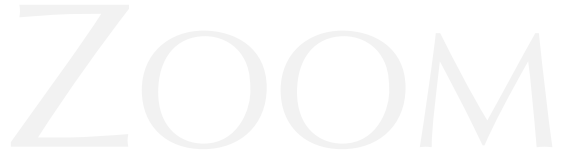

ORNA BEN-MEIR

Dans la vidéo La Mort de Molière, qui appartient à la période minimaliste de Wilson, on voit apparaître deux personnages déviants qui n'appartiennent pas à l'ambiance culturelle de l'œuvre, mais relèvent d'un autre royaume, celui de la nature. Je veux parler ici du renard, joué par un acteur, et du scarabée qui n'est qu'un accessoire de théâtre. Même s'il s'agit des seuls véritables personnages d'animaux visuellement présents dans l'œuvre, on peut y discerner d'autres représentations animales. On retrouve ainsi des animaux acoustiques, représentés par un fond sonore de rongements, d'aboiements, de cris et de gargouillis, ainsi que des animaux purement verbaux mentionnés dans six des séquences du texte de Müller: les poux (2), le chien (3), les vers (4), le requin et les baleines (5), le renard et le serpent (7), les oiseaux de proie et les bactéries (10). De nos jours, le chien est considéré comme un animal domestique, mais ce n'était pas le cas dans la France du XVIIe siècle ${ }^{1}$. Par ailleurs, les autres espèces animales mentionnées ne sont évidemment pas des animaux domestiques. Leur commun dénominateur est leur éloignement de la zone humaine française, soit parce qu'ils sont trop petits et trop dégoûtants, soit parce qu'ils sont trop grands et trop effrayants.

Une étude de l'œuvre de Wilson fait apparaître un élément frappant: la plupart de ses œuvres sont riches en images visuelles d'animaux, par exemple le décor représentant des pattes de chat géantes et le personnage de chien dans The King of Spain, la danse des autruches dans Life and Times of Joseph Stalin, le costume de panthère dans Death Destruction and Detroit II, et la gigantesque patte d'éléphant opposée aux petits rats dans l'installation Memory of a Revolution. La présence constante et significative de toute une faune dans ses œuvres laisse à penser que Wilson se sert de l'animal pour dire une chose dans les termes d'une autre, c'est-àdire pour représenter métaphoriquement ou symboliquement quelque chose qui n'est pas présent. La question de savoir ce qui se cache généralement derrière ce symbolisme animal dépasse les limites de cet article; je vais donc ici borner mon attention au symbolisme animal de la seule Mort de Molière, qui servira peut-être d'amorce à une recherche plus vaste.

Molière est un autre de ces nombreux héros qui forment la longue procession des personnages historiques dont se sert Wilson. Il s'agit de figures historiques 
dont les vies ont atteint une dimension mythique et parmi lesquelles on trouve Einstein, Edison, Staline, Freud, Napoléon, Bell, la reine Victoria et Frédéric le Grand. Dans La Mort de Molière, Wilson et Müller ont choisi de dépeindre ce génie du théâtre par les attributs physiques les plus bas. Müller le présente comme suit: «Molière c'est la puanteur de Versailles / [...] / Molière ce sont les pets dorés du roi soleil / Molière c'est l'odeur de sueur des comédies-ballets" (section 1). Structure en forme d'oxymore qui oppose les réactions animales sans contrainte du corps au comportement policé de la brillante cour du RoiSoleil. Le portrait visuel que donne Wilson de Molière est lui aussi fait d'images du corps, à part l'unique phrase: «Father, I am dying» répétée six fois au début de la cinquième séquence sur un constant fond sonore de rongements. Comme il se trouve cloué à son lit de mort, le langage corporel de Molière se limite à des mouvements des mains et de la tête. Bien que ce soit étrange et peut-être inclassable, il peut s'agir là d'un rappel de ce fait souvent oublié que Molière avait subi une formation de mime dans sa jeunesse. Dans le contexte du rationalisme cartésien du XVIIe siècle, la gestuelle était considérée comme un système de symboles purement accessoire et foncièrement barbare ${ }^{2}$. De plus, ces gestes qui appartiennent au répertoire de la farce - les mains qui grattent et qui repoussent, la bouche qui grogne, hurle et gronde - signifient encore aujourd'hui une perte de contrôle du corps et de l'esprit. Du temps de Molière, où la théorie cartésienne des "animaux-machines» considérait les animaux comme des automates dépourvus de sensibilité ${ }^{3}$, de tels comportements impliquaient une idée de bestialité. Ainsi le fait de représenter Molière dans un état proche de l'animalité reflète la force brute de son tempérament, une donnée biographique largement méconnue, mais qui se trouvait déjà soulignée dans la pièce de Boulgakov. Qu'est-ce qui se cache derrière une telle représentation? Wilson a déjà cité Einstein se décrivant lui-même comme «un homme bien ordinaire» et a remarqué ailleurs que ce qui l'impressionnait le plus dans la personne de Freud c'est qu' «il était... intensément ordinaire» ${ }^{4}$. S'agit-il seulement du désir de Wilson de faire descendre Molière de son piédestal? Ou ce recours à la présence effective d'animaux ne révèle-t-il pas une tout autre intention?

Pour répondre à ces deux questions, il convient de considérer le renard et le scarabée, tels qu'ils apparaissent à la fois visuellement et verbalement dans la vidéo, et de déterminer leur statut à la lumière de l'iconographie occidentale. Le renard et le scarabée sont des symboles animaux très riches qui relèvent d'une longue tradition iconographique. Le renard apparaît pour la première fois dans la troisième séquence de la vidéo, après le célèbre passage tiré de As You Like It. Un cadre gris neutre apparaît et la caméra bouge horizontalement jusqu'à ce qu'elle s'arrête sur la tête réaliste d'un renard. Il est en train de tendre l'oreille derrière le lit de mort de Molière, exactement comme le diable est montré épiant les lits de mort des chrétiens dans les images de l'Ars Moriendi ${ }^{5}$. L'assimilation du renard au diable est chose courante dans l'histoire de l'art, et d'une façon qui n'est guère surprenante, son image s'accompagne ici d'une citation en voix off du diabolique Dom Juan de Molière:

$$
\begin{aligned}
& \text { [...] un enragé, } \\
& \text { un chien, } \\
& \text { un diable, } \\
& \text { un Turc, } \\
& \text { un hérétique, } \\
& \text { qui ne croit } \\
& \text { ni Ciel, } \\
& \text { ni Enfer, } \\
& \text { ni loup-garou [...] }
\end{aligned}
$$

Il semble donc raisonnable de lire ce renard comme une extension de Molière lui-même occupé soit à s'observer lui-même en train de jouer son dernier rôle dans Le Malade imaginaire, soit en train de regarder Wilson en train de jouer la scène de sa mort. M'autorisant de l'opinion fréquemment avancée selon laquelle l'ambiguité est partie intégrante de l'esthétique de Wilson, je propose de considérer que 
ces deux interprétations autoréférentielles coexistent dans l'œuvre.

Cette lecture du renard est soulignée encore davantage dans la septième séquence. Cette scène, en effet, s'ouvre sur le même cadre gris avec un élément vertical placé en son milieu. La voix off récite un poème de l'artiste dadaïste et surréaliste Jean Arp:

Où dort mon renard en hiver?

Où se terre mon serpent?

Tout cela évoque, bien entendu, Jean de la Fontaine, le grand poète de l'époque de Molière, surtout connu pour ses fables mettant en scène des animaux et traitant la cour française comme une nation de caméléons. La caméra bouge à la verticale, révélant un poteau de bois et un acteur vêtu d'un pyjama gris qui laisse voir une partie de son torse nu, pieds nus et portant le masque du renard. Le renard de La Fontaine, puisque c'est bien lui, comme le confirme l'introduction de Linders 7 , est d'abord vu assis sur une chaise puis marchant vers le poteau, à quatre pattes comme un quadrupède, tournant autour du poteau et finalement y grimpant. L'acteur démontre ainsi tout le spectre des mouvements qui vont du pôle humain au pôle animal.

Il est typique des fables mettant en scène des animaux, y compris celles de La Fontaine, qu'elles montrent des animaux qui parlent, accordant ainsi à l'animal la seule capacité qui sépare la zone zoologique de la zone humaine: le langage. De plus, les animaux de La Fontaine ont ceci de particulier qu'ils se servent de leurs capacités linguistiques comme de tactiques permettant d'assurer leur survie physique. Certains animaux, par conséquent, se trouvent définis de façon stéréotypée par l'usage spécifique qu'ils font du langage. Ainsi le renard est-il caractérisé à l'époque comme le flatteur qui place le destinataire de ses paroles dans la position de légitimer et d'authentifier l'opinion de lui que l'autre lui manifeste ${ }^{8}$. À la lumière de la critique subversive que fait La Fontaine, dans ses fables, de la personnalité de Louis XIV, et de la description que fait Boulgakov des flatteries de Molière à l'endroit du roi, la vidéo rend évidente l'interprétation du renard comme une représentation du comédien du roi parvenant, grâce à ses talents langagiers, à circonvenir le Roi-Soleil en l'enrôlant comme participant actif dans les spectacles de la cour.

La voix off, que l'on entend dans la scène du renard, fait la lecture d'une lettre envoyée à Wilson par le critique de danse Edward Denby, qui lui recommande de prendre garde à sa toux. Le rapport qu'établit Denby entre cette toux et la mort, mais aussi le diable, fait venir à l'esprit du spectateur le renard diabolique de la troisième séquence et réaffirme la relation autoréférentielle entre Molière et celui qui joue son rôle, Wilson lui-même. Molière souffrait d'une maladie pulmonaire qui, vers la fin de sa vie, rendait son jeu plus difficile en lui infligeant la torture d'une toux incontrôlable. Il se servait de cette toux en en faisant un attribut de ses personnages, assimilant ainsi l'acteur à l'homme. Ainsi la façon dont Wilson se sert d'une lettre personnelle concernant sa toux évoque-t-elle la façon dont Molière incorporait sa toux réelle à son art. En outre, la toux de Molière fait aussi penser à ses autres défauts de langage - sa voix sourde, la dureté de ses inflexions, son débit trop rapide, ses hoquets -, un rappel de l'intérêt que porte Wilson au langage et qui vient de ses propres problèmes quand il était enfant. Il existe d'autres points de ressemblance entre les deux hommes, comme leur éducation très bourgeoise, leurs pères autoritaires et le fait que tous deux ont renoncé à une profession honorable et au droit de succéder à leurs pères pour se lancer dans une carrière théâtrale pour le moins aventureuse. Ils partagent aussi une forme de comportement animal: Wilson avait l'habitude d'émettre des grognements ou des aboiements de chien, en particulier après avoir reçu son congé d'un hôpital psychiatrique ${ }^{9}$. Il s'est sans doute beaucoup identifié au personnage de Molière dont les frontières floues qu'il ménageait entre sa vie et la scène trouvent leur plus forte illustration dans les circonstances de sa mort ${ }^{10}$.

Il est plutôt surprenant qu'un acteur affligé des difficultés de langage dont souffrait Molière ait pu prospérer dans la seconde moitié du dix-septième 
siècle, car cette époque regardait l'habileté langagière comme la marque de l'homme intelligent et civilisé par opposition à l'homme animal, l'homme de la nature, qui en était souvent dépourvu. Dans ce tempslà, le langage n'était pas considéré comme un moyen de véhiculer les pensées et les intentions de l'émetteur, mais comme une carte d'entrée dans la société savante 11 et le fondement même du pouvoir et du contrôle. On déguisait ses motifs, ses craintes et ses pulsions personnelles en les traduisant dans un langage et des idéologies codées. En reconnaissant que l'ordre linguistique régente son univers, Molière, comme le renard de La Fontaine, exploite le langage d'une façon inédite: sa principale stratégie consiste à construire des situations comiques en suscitant des interférences dans le processus normal de la communication et en libérant le langage grâce à de brusques changements dans les façons de parler. C'est dans Les Femmes savantes, où le thème central est le langage, que Molière déploie le plus son exceptionnelle élégance verbale. Müller en a choisi des extraits pour la huitième séquence, qui fait appel à un dialogue en voix off entre la morte Madeleine Béjart, le grand amour de la vie de Molière, et Armande, fille ou sœur de Madelaine et femme de Molière.

Les deux femmes font une veillée mortuaire au chevet de Molière. La complexité de la relation qui les unit est visuellement représentée par la somptueuse robe d'époque rouge vif et la coiffure également d'époque d'Armande, qui contrastent avec la robe et le voile blanc transparent tout simples que porte, tel un linceul, Madeleine. Les paroles de Madeleine sonnent comme une voix off jusqu'au moment où elles deviennent indistinctes. Son voile se soulève et l'on voit alors un insecte bleu-vert logé dans sa bouche ouverte. Un gros plan permet de l'identifier de façon précise comme étant le Scarabaeus sacer, ou bousier. Madeleine ôte le scarabée de sa bouche et le dépose sur le dos de la main droite de Molière, un geste qui évoque visuellement à la fois l'imposition d'un sceau et l'offrande d'une amulette funéraire. Le bousier est le symbole visuel du célèbre scarabée de l'ancienne Égypte, qui donnait des pouvoirs divins à celui qui le portait, parce qu'il était considéré comme l'incarnation du Dieu créateur fusionné au dieu soleil et au pharaon. Le scarabée ainsi transformé en amulette sacrée signifie ici le pouvoir de droit divin du Roi-Soleil étendant sa protection sur la vie de Molière. C'est cette protection qui pourrait expliquer, si la chose est vraie, l'audace du comportement incestueux de Molière épousant sa propre fille.

Mais l'analogie entre le bousier et le Dieu créateur, qui se fonde sur l'idée erronée entretenue par les Égyptiens que le jeune scarabée émergeant de la bouse était le fruit d'un acte d'auto-engendrement, pourrait bien nous fournir la clé d'une autre interprétation. Il peut, en effet, être interprété comme une représentation de Molière, créateur de lui-même faisant de sa vie une véritable création. C'est ce que souligne le texte extrait des Femmes savantes, ce dialogue fort spirituel entre deux jeunes sœurs déterminées qui ouvre la pièce, où la plus vieille des deux sœurs, Armande, et la plus jeune, Henriette, toutes deux également en âge de se marier, parlent de transformer la vie en une création. Comme Madeleine Béjart et sa fille Armande, les deux sœurs sont des rivales qui se disputent le même homme, Clitandre. Mais Armande, qui représente la femme savante ${ }^{12}$, exprime la répulsion physique que lui inspire le mariage - «Soumettant à ses lois la partie animale» et souligne les plaisirs sensuels de l'esprit et de l'intelligence, alors que sa sœur Henriette, qui elle n'est pas savante, lui oppose des arguments intellectuels en faveur des désirs physiques dont le mariage est l'exutoire. Toutes deux parlent de création: Armande évoque la création de l'esprit et Henriette loue celle du corps. En ce sens, le dramaturge, en tant que manipulateur de mots est l'égal de la mère qui donne la vie et dont le rejeton est décrit en termes purement matériels. Cette dichotomie culture/nature, fondamentale au temps de Molière, culmine dans un paradoxe: le mariage entre les sphères élevées de la recherche intellectuelle et les bas appétits charnels, un mariage que structure le signe linguistique qui distingue l'homme de l'animal. Soit, dans les mots de Descartes: 
Le langage est en effet le seul signe certain d'une pensée latente dans le corps; tous les hommes en usent [...] mais aucune bête ne peut en user; c'est pourquoi il est permis de prendre le langage pour la vraie différence entre les hommes et les bêtes. 13

Il semblerait donc que la zone zoologique, sur laquelle nous avons opéré rapidement un zoom-in et un zoom-out, n'est pas seulement la somme des symboles qui la constituent, dans le cas qui nous occupe le renard et le bousier, mais représente aussi un métasymbole dans l'œuvre de Wilson. Car à travers le symbolisme animal, Wilson ne se contente pas de représenter l'homme Molière et son époque, ou n'importe lequel des personnages historiques dont il se sert dans son œuvre, mais exprime plutôt sa préoccupation artistique fondamentale pour le visuel. En effet, au contraire des fables de La Fontaine, les fables de Wilson mettent en scène l'animal sans parole et surtout l'animal-homme, en exigeant du spectateur qu'il «écoute» ses images visuelles comme si c'étaient de simples mots composant son écriture du corps. Cette poétique visuelle renverse la tyrannie du langage à l'œuvre dans la pensée occidentale et que représente Descartes. Parallèlement à cette exigence de Wilson, le philosophe Jacques Derrida demandera à son lecteur de «visualiser» le texte:

MIMIQUE, ou plutôt $m i+m i+q u e$, c'est-à-dire deux fois les moitiés plus l'indication ou l'intimation subjonctive de la subordination mimée; mi-mais? Mais-qui? Mimi à que(ue)? Queue de mémé? 14

Wilson et Derrida souhaitent tous deux libérer les signes de notre culture, qu'ils soient visuels ou verbaux, de leur subordination traditionnelle aux signifiés.

\section{NOTES}

1. L'entretien d'animaux domestiques est une pratique récente, surtout répandue dans les milieux bourgeois urbains. À ce sujet, cf. A.-H. Maehle, "Cruelty and kindness to the "Brute Creation". Stability and change in ethics of the man-animal relationship, 1660-1850", dans Animals and Human Society, A. Manning and J. Serpell (sous la dir.), London \& New York, Routledge, 1994.

2. Cf. S. Releya, Signs, Systems and Meanings. A Contemporary Semiotic Reading of Four Molière Plays, Connecticut, Wesleyan University Press, 1976, p. 5 .

3. Cf. Maehle, note 1.

4. E. Levy, "Robert Wilson: Theater History, Theater as History", Parkett, 16, 1988, p.66-69.

5. Il s'agit d'un célèbre ouvrage de piété du XVe siècle, illustré de gravures et originaire de l'Europe du Nord.

6. Extrait de la célèbre tirade de Sganarelle, à la scène Ire de l'acte Ier (N.D.T.).

7. J. Linders, «Molière \pm Müller», Performance Research, vol. I, nº 2, 1996, p. 93-95.

8. Une excellente analyse d'une fable mettant en scène le renard permet à Louis Martin de bien illustrer l'atmosphère particulière de cette époque. Cf. "The Fox's tactics", dans Portrait of the King, London, Macmillan Press, 1988.

9. Cf. J. Johnston, «Family Spectacles", Art in America, vol. 74, décembre 1986, p. 94-107.

10. Dans la note 8 de l'article mentionné, Johnson signale déjà cette identification à Molière.

11. En francais dans le texte (N.D.T.).

12. En français dans le texte (N.D.T.).

13. R. Descartes, Euvres et Lettres, Paris, Gallimard, coll. «Bibliothèque de la Pléiade ", 1953; Méditation III, p. 1320.

14. J. Derrida, La Dissémination, Paris, Éd. du Seuil, coll. «Tel Quel », 1972, p. 257. 


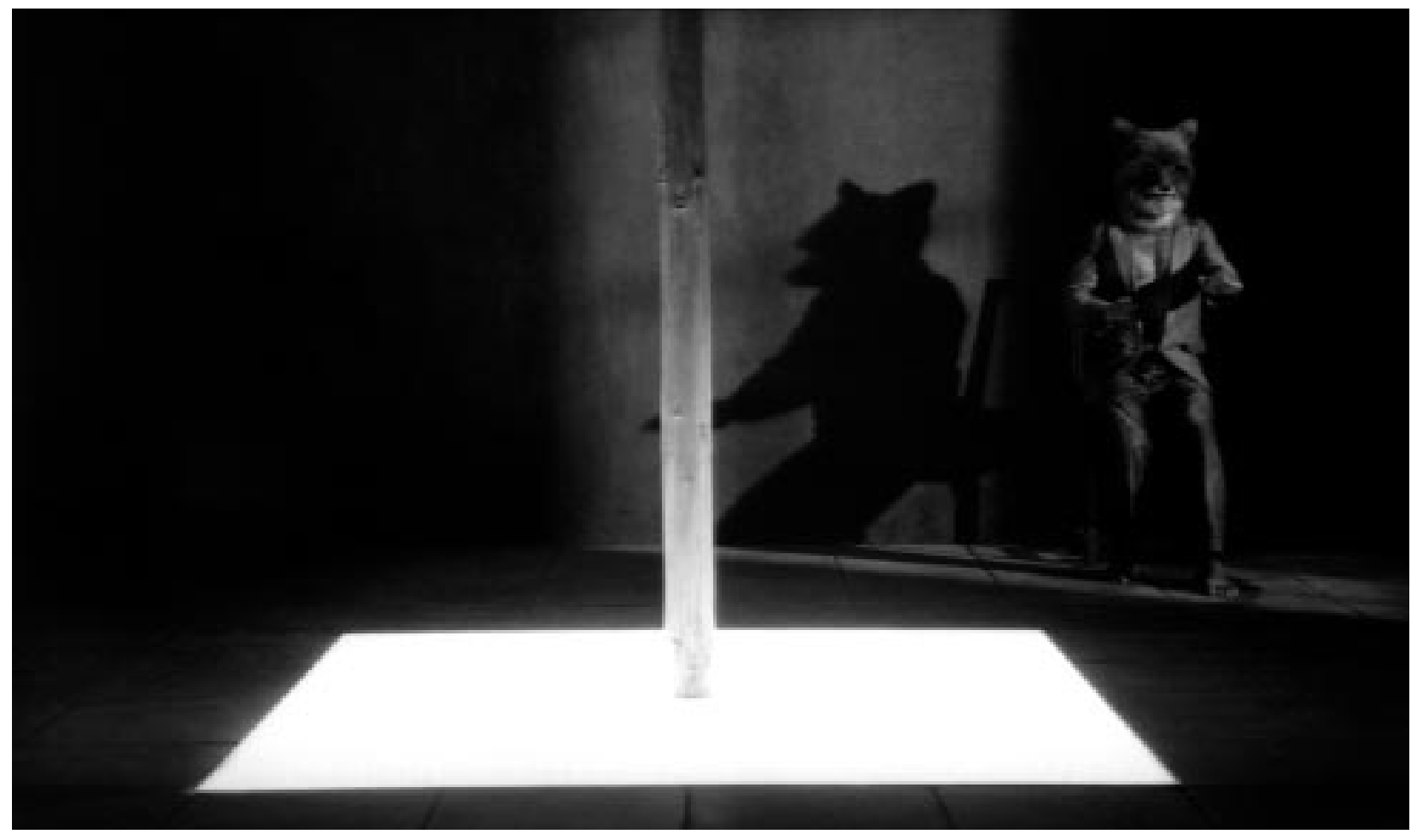

La Mort de Molière de Robert Wilson. Photo INA. 